\title{
Analysis of the velocity diagrams of impellers of centrifugal compressor stages after the preliminary design
}

\author{
Aleksandr Drozdov ${ }^{1}$, Alexey Rekstin ${ }^{1, *}$ \\ ${ }^{1}$ Peter the Great Saint Petersburg Polytechnic University, Joint Institute of Science and Technology, \\ Scientific-Research Laboratory “Gas dynamics of turbomachines”, Polytechnicheskaya, 29, 195251, \\ Saint Petersburg, Russia
}

\begin{abstract}
Preliminary design is an important stape in the development of centrifugal compressors and compressor stages. Basically for this purpose, various recommendations on the choice of the flow path dimensions are applied. Researchers of the Research and Development Laboratory "Gas dynamics of turbomachines" prof. Yu.B. Galerkin and A.F. Rekstin analyzed and summarized the dimensions of flow paths of 124 impellers. On the basis of this analysis, formulas were proposed for choosing the flow path dimensions of the centrifugal compressor stages, which were included in the preliminary design program. The formulas used are designed for relative Mach number of 0.7 and isentropic coefficient of 1.4. The correct application of these formulas for other Mach numbers and isentropic coefficient required development of an appropriate approach and algorithm for adjusting the height of the impeller blades at the outlet. Calculations of gas-dynamic characteristics using the Universal Modeling Method showed the need for selecting a coefficient that takes into account the influence of viscosity to obtain the required pressure characteristics of the compressor stage. This problem was also solved in the program of preliminary design. To check the quality of preliminary design, the results were verified using a non-viscous quasi-three-dimensional calculation program. Three stages were designed for parameters different to those used for development of preliminary design formulas. Analysis of the velocity diagrams of the impeller blades and distribution of meridional velocities showed good results of the preliminary design.
\end{abstract}

\section{Nomenclature}

$\bar{b}_{1}$ is blade height at the inlet; $\bar{b}_{2}$ is blade height at the outlet; $D_{2}$ is impeller diameter; $\bar{D}$ is relative diameter; $\overline{\mathrm{D}}_{\text {hub }}$ is relative hub ratio; $k$ is isentropic coefficient; $K_{p c}$ is coefficient of the pressure center position of the velocity diagram; $\bar{L}_{r s}$ is position of the leading edge of the impeller on the periphery; $\bar{L}_{r h}$ is position of the leading edge on the hub; $\bar{L}_{m}$ is relative axial length; $M_{u}$ is relative Mach number; $\bar{R}_{S}$ is relative shroud radius; $\bar{R}_{h}-$ is relative hub radius;

\footnotetext{
*Corresponding author: rekstin2k7@mail.ru
} 
$z_{\text {im }}$ is the amount of impeller blades; $\Phi_{\text {des }}$ is design flow rate coefficient; $\psi_{\text {Tdes }}$ is design loading factor; $\psi_{\text {Tid }}$ is loading factor at inviscid flow; $\gamma$ is the angle of the entrance edge of the blade; $\varphi_{2}$ is angle of inclination of the shroud; $\beta$ is the angle between relative velocity and reverse circumferential direction; $\beta_{b l}$ is the blade angle; $\bar{\delta}_{b l}$ is relative blade thickness; $\rho$ is gas density.

\section{Subscripts:}

$0,1,2$ are indices of control sections; des is referring to the design flow rate.

\section{Superscripts}

* refers to total parameters.

\section{Introduction}

Centrifugal compressors are used in various industries. The widest field of application of centrifugal compressors in Russia is the extraction and transportation of natural gas. The main part of gas industry compressor park consists of centrifugal compressors, which are used to transport gas through pipelines and to inject gas into underground storage facilities. The active development of new gas fields, development of the country's gas transmission system generates a constant demand for centrifugal compressors. Many currently used compressors are morally and physically obsolete. This leads to the need to replace the gaspumping units as a whole or, more effectively, to replace the flow path in the existing housing.

A large amount of energy is expended on drive of compressors; therefore, the gasdynamic design of centrifugal compressors [1-9] is still an important and urgent task.

Modern design of new compressors is based on the use of developed and previously tested model stages, as well as on the use of specialized engineering techniques. The development of mathematical models that underlie these techniques is carried out by specialists from universities and specialized enterprises [10-20].

For decades scientists from SPbPU have been researching turbo compressors. The obtained results allowed us to create a gas-dynamic calculation method for centrifugal compressors, an appropriate mathematical model and a software package based on it, called the Universal Modeling Method.

In the first versions of the Universal Modeling Method [21 - 26], preliminary design is based on qualitative recommendations from classical Russian monographs and on our own research [27 - 31].

The program "Optimal Design of a Centrifugal Compressor Stage” is a part of the Universal Modeling Method which considers the preliminary design of stage composed basing on the recommendations of the general nature. The following characteristics are calculated using mathematical models:

- mode of non-incidence flow of the impeller, which is taken as the design mode of stage;

- loading factor on the design mode;

- efficiency.

By an automatic search through the main dimensions the program determines their combination, in which a non-incidence flow is achieved at a flow rate $\Phi_{\text {calc }}$ set for a stage, and a given loading factor $\psi_{\text {Tcalc }}$ is provided with the maximum possible efficiency. The program "Calculation of the family of centrifugal compressor stage characteristics" of the Universal Modeling Method performs this for an optimized version of the flow path. But, 
since mathematical models based on empirical coefficients cannot guarantee the absolute reliability of the calculated characteristics, the user of the program should analyze the correctness of the optimized dimensions, based on the experience of the design and the results of testing the stages and compressors. The described approach requires a great deal of personal experience in design and research work from the user.

Industrial partners which are potential users of the Universal Modeling Method, raised the question of adjusting the design procedure in such a way that reliable provision of a given flow rate and compressor head could be provided by a project composed by a wide range of users.

\section{Method}

Researchers of the laboratory "Gas Dynamics of Turbomachines" under the leadership of prof. Yu.B. Galerkin selected the following solution to the stated problem: transition from preliminary design on the basis of qualitative recommendations to design on the basis of specially conducted studies and approximating equations that give specific values for each of the dimensions of the flow path. In [32], such equations were proposed based on the results of designing two series of model stages in the range of flow coefficients $\Phi_{\text {des }}=0.015-0.15$. The equations are used optionally in the program "Calculation of the family of centrifugal compressor stage characteristics". The trial operation of the program as a design program showed the rationality of the approach, but with regard to the choice of dimensions that provide the required values of $\Phi_{\text {des }}$ and $\psi_{\text {Tdes }}$, the problem was not solved. In [33], the question was studied in relation to low-flow stages, for which the techniques of preliminary design are poorly understood.

The method of research is the analysis of velocity diagrams of the impellers blades streamlined by an inviscid quasi-three-dimensional flow. The monographs [27, 28] showed that the calculation of diagrams using the 3DM.023 program, which uses a combination of the quasi-orthogonal method and integral equations, gives reliable results for calculating the work coefficient of a real impeller and determining the mode of non-incidence flow, i.e. to determine the design mode of the impeller.

Using the analysis of velocity diagrams, A. Rekstin and prof. Yu. Galerkin designed 124 impellers in the range of gas-dynamic parameters $\Phi_{\text {des }}=0.015-0.15, \psi_{\text {Tdes }}=0.40-0.70$ with hub ratios in the range $\overline{\mathrm{D}}_{\text {hub }}=0.25-0.40$.

Figure 1 shows a meridional form of the flow path of 3D and 2D impellers; dimensions of the flow path are indicated. 

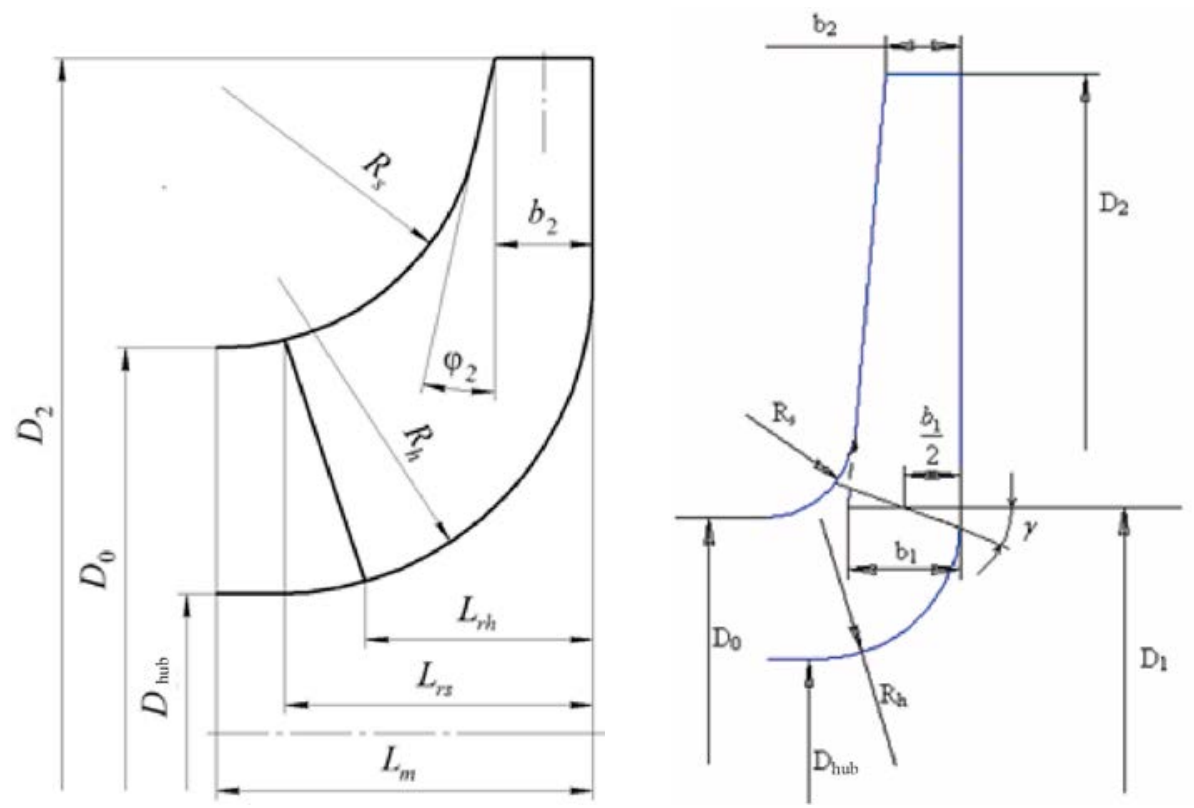

Fig. 1. Scheme and dimensions of impeller in meridional plane. 3D impeller is on the left, 2D impeller is on the right.

\subsection{Aim of the work}

The purpose of this work is to implement the proposed by A.F. Rekstin and prof. Yu.B. Galerkin algorithm in a computer program of preliminary design and to verify the program within the studied design parameters $\Phi_{\text {des }}=0.015-0.15, \psi_{\text {Tdes }}=0.40-0.70, \overline{\mathrm{D}}_{\text {hub }}=0.25$ -0.40 .

The algorithm proposed by A. Rekstin and prof. Yu. Galerkin should be supplemented with respect to the choice of the relative height of blades. The formulas recommended by them give the value of the relative height of blades $\bar{b}_{2}=f\left(\Phi_{\text {des }}\right)$, which provide the specified loading factor under the similarity criteria $M_{u}=0.70, k=1.4$. With a greater increase in density at the exit of impeller, the relative height of the blades should be reduced, and vice versa

$$
\begin{gathered}
\psi_{T}=1-\varphi_{2} \times \operatorname{ctg} \beta_{2} ; \\
\varphi_{2}=\frac{\Phi}{4 \bar{b}_{2}} \frac{\rho_{0}^{*}}{\rho_{2}} .
\end{gathered}
$$

The particular aim of the work is creation of a method for calculating the relative height of blades, which provides the specified loading factor $\psi_{\text {Tdes }}$ with $M_{u} \neq \neq 0.70, k \neq 1.4$.

\subsection{Method of calculation the relative height of blades at the exit for arbitrary values of compressibility criteria}

To calculate the height of blades at the exit of the impeller $\bar{b}_{2}$, which provide the necessary value of $\psi_{\text {Tdes }}$ at $M_{u} \neq 0.7$ and $k \neq 1.4$ we use mathematical models of the Universal Modeling Method.

At the first stage of preliminary design according to the formulas of A.F. Rekstin and prof. Yu.B. Galerkin the value $\bar{b}_{2}$ is calculated, which ensures that the given $\psi_{\text {Tdes }}$ is obtained when the Mach number $M_{u}=0.7$ and the isentropic coefficient $k=1.4$. 
A.F. Rekstin and prof. Yu.B. Galerkin for the calculation of $\psi_{\text {Tdes }}$ used the loading factor for non-viscous flow with an empirical coefficient. The preliminary design program includes a head model based on the proposed by prof. Yu.B. Galerkin formula [28] with an empirical coefficient $K_{\mu}$, taking into account the influence of viscosity [27]:

$$
\psi_{\text {Tdes }}=\frac{1-\varphi_{2}^{\prime} \operatorname{ctg} \beta_{b l 2}}{1+\frac{K_{\mu} \sin \beta_{b l 2}}{z_{\text {im }} K_{p c}\left(1-\bar{D}_{1}\right)}} .
$$

For the stage developed by the preliminary design formulas, it is necessary to search for the value $K_{\mu}$ that provides the given $\psi_{T \text { des }}$. For this, the fractional steps method is used for searching the desired $K_{\mu}$ value upward or downward from the current one. At the end of the article, examples of preliminary stage design with $M_{u} \neq 0.70, k \neq 1.4$ are given. The specified $\psi_{\text {Tdes }}$ values are provided by appropriate selection of the relative blades height.

\subsection{The preliminary design program}

The preliminary design program "Preliminary design and calculation of the family of centrifugal compressor stages characteristics " according to the developed method was made on the basis of the 6th version of the program "Calculation of characteristics family for centrifugal compressor stages" (7th version of the Universal Modeling Method is focused on designing trans- and supersonic compressors [34 - 36]). The program of preliminary design has retained the functions of its predecessor and can operate in three modes:

- calculation of the stage characteristics with dimensions entered by the user;

- calculation of the stage characteristics with dimensions obtained in the optimal design program based on mathematical models. The dimensions and parameters of the optimal variant are automatically transferred to the program for calculating the family of characteristics;

- the dimensions and shape of the flow path are calculated according to the developed preliminary design method. The preliminary design of impellers is carried out according to the method that summarizes the results of researching the parameters 124 of optimized impellers. Stator elements are calculated by the method from [32].

Figure 2 on the left shows the parameter entry menu for calculating the gas-dynamic characteristics. 


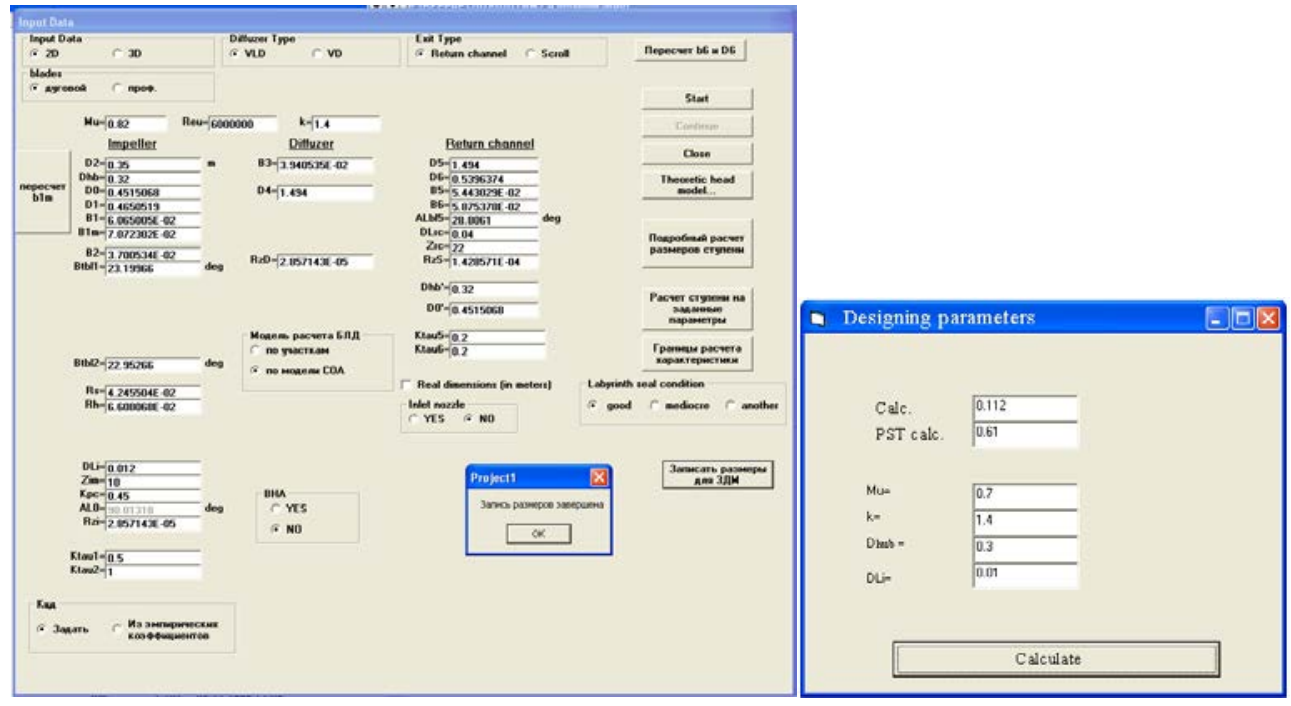

Fig. 2. The program "Preliminary design and calculation of a family of characteristics of centrifugal compressor stages". The menu for inputting the step parameters for calculating the gas-dynamic characteristics (left). Menu for entering stage parameters for preliminary design (right)

The upper line of windows allows one to select the type of impeller, diffuser, outlet device. In addition to entering the criteria of similarity and size, there are a number of buttons for launching options that facilitate the calculation and allow one to select options for mathematical models of efficiency and head depending on the characteristics of the problem being solved. Figure 2 on the right shows the parameter input window for preliminary design.

\section{Results}

\subsection{Verification of the preliminary design method for impeller}

The choice of dimensions during the preliminary design is based on the parameters generalization for 124 impellers discussed above. These impellers have discrete values of design parameters:

$-\Phi_{\text {des }}=0.015 ; 0.028 ; 0.0346 ; 0.0526 ; 0.080 ; 0.0936 ; 0.1095 ; 0.128 ; 0.15$;

- $\psi_{\text {Tdes }}=0.40 ; 0.482 ; 0.581 ; 0.70$;

- $\overline{\mathrm{D}}_{\text {hub }}=0.25 ; 0.30 ; 0.35 ; 0.40(\mathrm{RI}) ; 0.25 ; 0.30 ; 0.35(\mathrm{AI})$.

Calculations of these impellers were carried out for the similarity criteria $M_{u}=0.70, k=$ 1.4 and blade thickness $\bar{\delta}_{b l}=0.12$. If the preliminary design meets the requirements for intermediate values $\Phi_{d e s}, \psi_{T d e s}, \bar{D}_{h u b}$, and $M_{u}, k$ and $\bar{\delta}_{b l}$ different from the abovementioned values, the method verification is successful.

\subsection{An example of preliminary design of low-flow 2D impeller}

The parameters of the low-flow 2D impeller 0.022-0.52-029 are evident from its name. The project was carried out at $M_{u}=0.56, k=1.32$ and blade thickness $\bar{\delta}_{b l}=0.012$. All design parameters are different from the parameters of 124 impellers discussed above. 
The initial parameters and dimensions of 2D impeller 0.022-0.52-029 are transmitted after the initial design to the program for calculating the velocity diagrams of an inviscid quasi-three-dimensional flow 3DM.023 and the calculation is performed.

Figure 3 shows the result of calculations in the 3DM.023 program.

When designing the above 124 impellers, the provision of the given value of $\psi_{\text {Tdes }}$ was checked by the value of the loading factor for the inviscid flow $\psi_{\text {Tid }}$. Its designation in Figure 3 is "PSTid". According to the analysis of the characteristics of the model stages of the 20CE family [37], when designing an impeller, the empirical relation $\psi_{\text {Tdes }} / \psi_{\text {Tid }}=0.93$ was adopted. For 2D impeller 0.022-0.52-029, the $\psi_{\text {Tid }}$ value should be equal to 0.559 . It turned out to be 0.55 . That is, in the preliminary design the shortage of the loading factor is a small value of $1.6 \%$. This can be offset by the final optimization of the flow path.

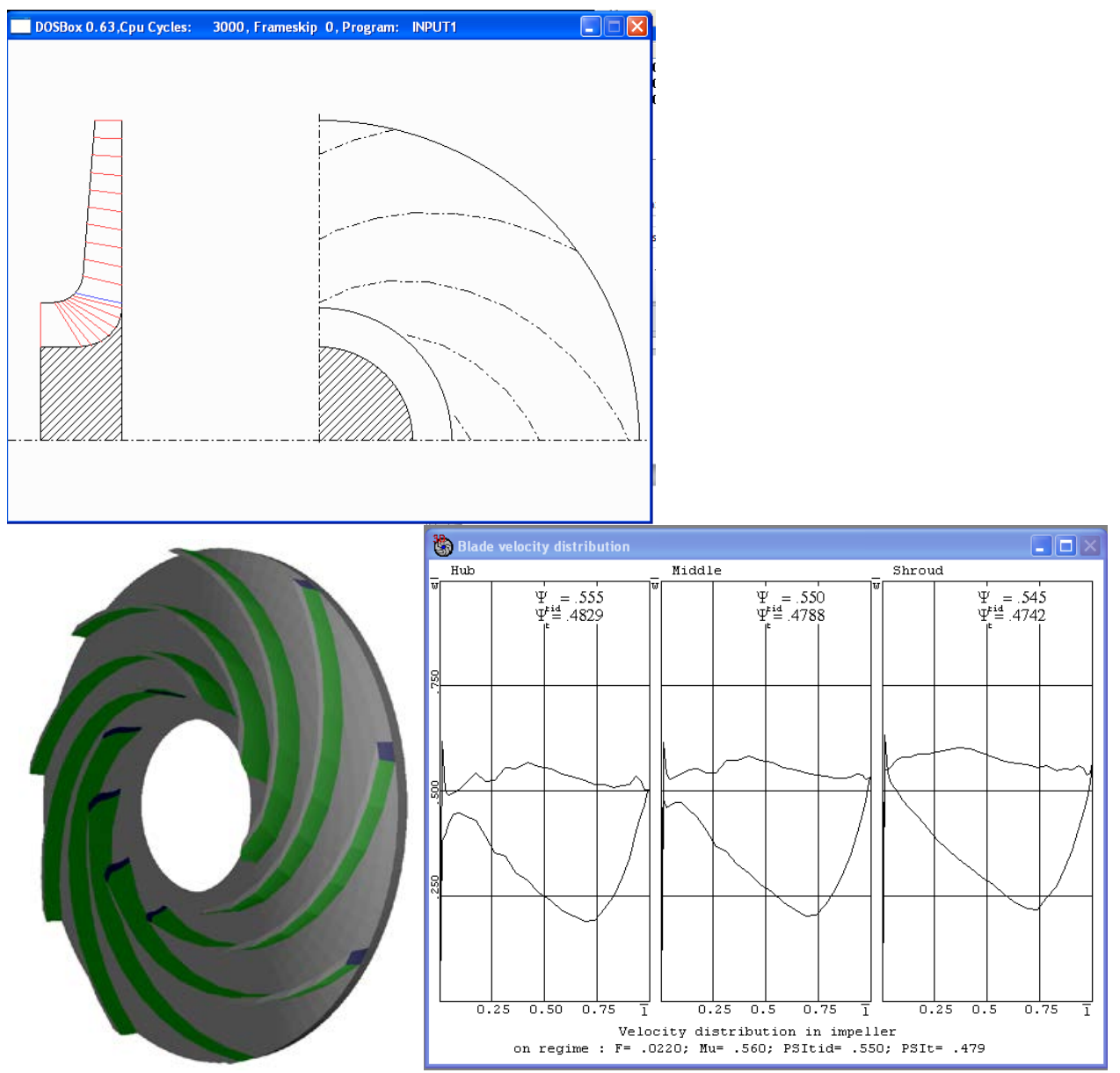

Fig. 3. Program 3DM.023. Scheme of the flow path of RI 0.022-0.52-029, blade profile and velocity diagram for the three current surfaces

\subsection{An example of preliminary design of mid-flow 2D impeller}

The parameters of the mid-flow 2D impeller 0.077-0.63-038 are evident from its name. The project was carried out at $M_{u}=0.83, k=1.55$ and blade thickness $\bar{\delta}_{b l}=0.010$. All design parameters are different from parameters of 124 impellers discussed above. 
Figure 4 shows the flow path view, blade profile and velocity diagram on three current surfaces of the 2D impeller 0.077-0.63-038, which is data from the 3DM.023 program.

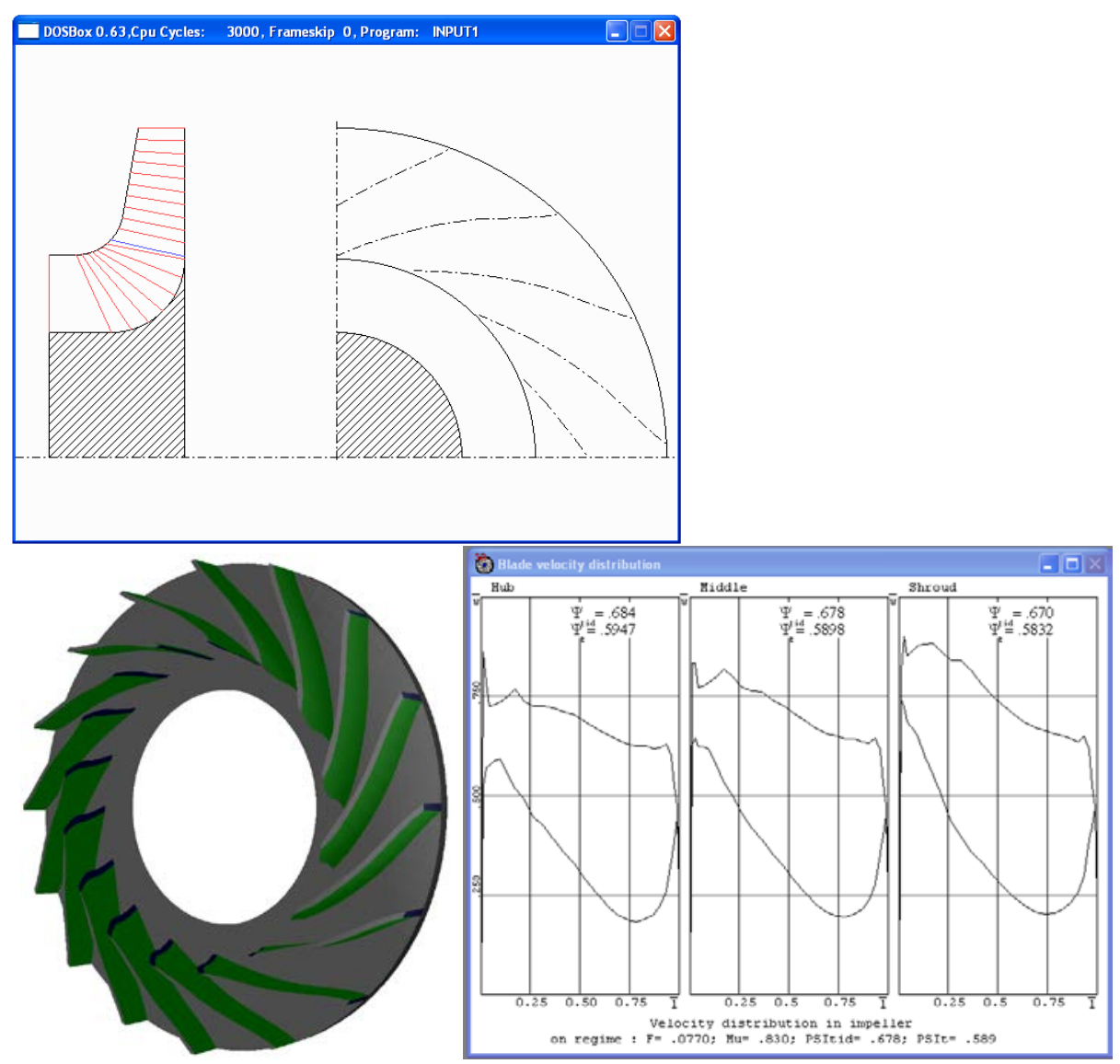

Fig. 4. Program 3DM.023. Scheme of the flow path of 2D impeller 0.077-0.63-038, blade profile and velocity diagram for the three current surfaces

\subsection{An example of preliminary design of high-flow 3D impeller}

The 3D impeller 0132-0.545-0265 was designed at $M_{u}=0.90, k=1.60$. The design parameters and blade profile are presented in Figure 5. 


\section{EECE-2018}

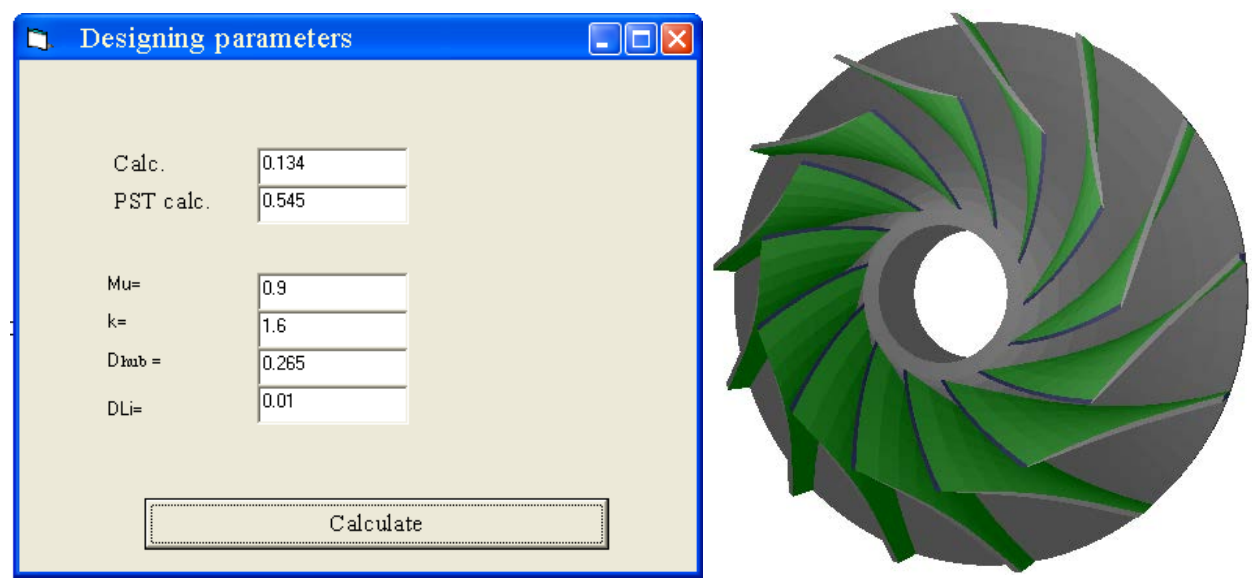

Fig. 5. Impeller AI 0132-0.545-0265. Designing parameters and blade profile

Figure 6 on the left shows the velocity diagrams for 3D impeller 0132-0,545-0265 based on results of the preliminary design. On the right we present velocity diagrams after minor adjustments.

The type of velocity diagrams corresponds to the design principles, but the ratio $\psi_{\text {Tdes }} / \psi_{\text {Tid }}=0.545 / 0.596=0.914$ indicates a slight excess of the loading factor. A small negative incidence angle on the shroud can be reduced. To obtain correction, it was sufficient to reduce the number of blades by $1 \mathrm{pc}$. and increase degree the inlet angle of blades by one. The ratio $\psi_{\text {Tdes }} / \psi_{\text {Tid }}=0.545 / 0.589=0.925$ is slightly different from the value of 0.93 , adopted at the optimal design.
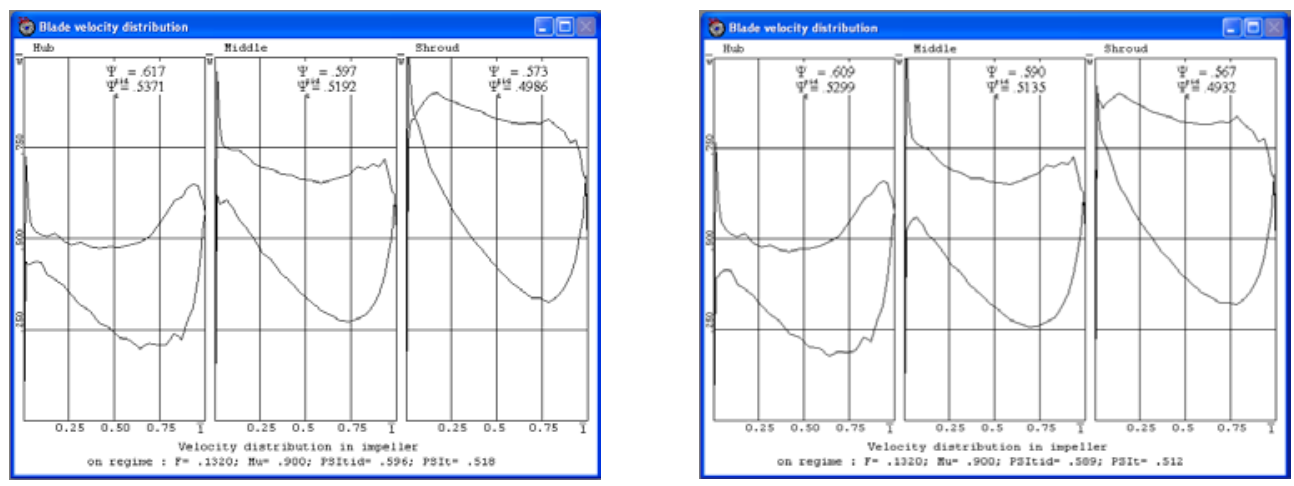

Fig. 6. Program 3DM.023. On the left are velocity diagrams for 3D impeller 0132-0.545-0265 based on the results of the preliminary design. On the right are velocity diagrams after minor adjustments.

\section{Results and discussion}

The proposed methodology for the preliminary design of centrifugal impellers was tested and the design results were compared with the calculations for the inviscid quasi-threedimensional calculation program. Analysis of the velocity distribution diagrams along the surfaces of impeller blades showed that the dimensions and shape of the flow path of impeller, obtained as a result of the design, ensures its efficient operation. Both 2D and 3D impellers were calculated with different design flow coefficients and loading factors. This provided an effective test of the changes introduced to the mathematical model. 
Taking into account the fact that the proposed methodology of preliminary design does not imply at this stage an iteration search for optimal dimensions of the impeller flow path, the accuracy of calculations is comparable to more complex and time-consuming methods $[16,17]$.

The changes of the engineering program for designing centrifugal compressors (the Universal Modeling Method) has shown its efficiency and can find practical application in the implementation of new projects of centrifugal compressors.

\section{Conclusions}

The method of preliminary design proposed by A. Rekstin and prof. Yu. Galerkin was supplemented by the method of calculating the relative height of blades at the exit of impellers, providing a given loading factor in a wide range of compressibility criteria. Performed on the basis of these methods and the Universal Modeling Method, the program "Preliminary Design and Calculation of the Family of Characteristics of Centrifugal Compressor Stages” proved to be a reliable and convenient tool for gas-dynamic design.

The presented scientific research was performed at the Scientific-Research Laboratory "Gas dynamics of turbomachines" of SPbPU, headed by prof. Yu.B. Galerkin. The authors are grateful to prof. Yu.B. Galerkin for the problem statement and advices while performing the work.

The research was performed by a grant of Russian Science Foundation (project No. 18-79-10165).

\section{References}

1. V.E. Evdokimov Turbines and compressors. 2, 3-4 (1997)

2. A.N. Lyubimov, V.E. Evdokimov Compressors and pneumatics. 7, 28-33 (2012)

3. A.V. Semakov, V.E. Evdokimov, A.I. Reprintsev, A.N. Lyubimov Compressors and pneumatics. 5, 20-22 (2013)

4. A.N. Lyubimov, V.E. Evdokimov, A.V. Semakov, A.I. Reprintsev Compressors and pneumatics. 4, 12-20 (2014)

5. A.T. Lunev. Compressors and pneumatics. 10, 4-7 (2001)

6. A.T. Lunev Dis ... Cand. tech. sciences. p. 123 (2005)

7. K. Kabalyk, W. Kryłłowicz Transactions of the fluid-flow machinery 131, 41 - 53 (2016)

8. W. Kryłłowicz, P. iderwider, Z. Kozanecki, K. Kabalyk, Jr. Z. Kozanecki 12th European Conference on Turbomachinery, 45-52 (2017)

9. P. Harley, S. Spence, D. Filsinger, M. Dietrich, J. Early Journal of Turbomachinery 137 DOI: 10.1115/1.4028247 (2015)

10. T. Syka, O. Luňáček EPJ Web of Conferences 45 DOI: 10.1051/epjconf 20134501088 (2013).

11. P. Li, C. Gu, Y. Song Energies 8, p. 4317-4334; doi: 10.3390/en8054317 (2015)

12. R.H. Aungier Centrifugal Compressors: A Strategy for Aerodynamic Design and Analysis (2000)

13. M. Casey, C. Robinson Journal of Turbomachinery 135 (2013)

14. R.H. Aungier Transactions of the ASME 117, (1995)

15. Kangsoo Im Ph. D. thesis p. 160 (2012) 
16. H. Hazby, M. Casey, C. Robinson, R. Spataro Progress of 12th European Conference on Turbomachinery Fluid dynamics \& Thermodynamics ETC12 Paper ID: ETC2017-134 (2017)

17. D. Japikse, E.M. Krivitzky Proceedings of the ASME Turbo Expo DOI: 10.1115/GT2016-57956 (2016)

18. D. Japikse. JSME fluid engineering conference. p. 34 - 44 (2003)

19. C. Stuart, S. Spence, S. Kim, D. Filsinger, A. Starkee Proceedings of the international gas turbine congress pp. 485-494 (2015)

20. X. Gong, R. Chen Journal of Mechanical Engineering Research 4 (2), pp. 45 - 59 (2014)

21. Yu.B. Galerkin Compressors and pneumatics 2, 9-14 (2000)

22. Yu.B. Galerkin, K.A. Danilov, E.Yu. Popova Compressors and pneumatics. 2 (1993)

23. Yu.B. Galerkin, V.P. Mitrofanov Chemical and Petroleum Engineering V.31 № 11-12 (1996)

24. Yu.B. Galerkin Some problems of power engineering. p. 22-30 (1995)

25. Yu.B. Galerkin, E.Yu. Popova, K.A. Danilov. Works by German engineer. Germany. p. 54 - 59 (1995)

26. Yu.B. Galerkin Communications of the Union of German Engineers. 947 (1992)

27. Yu.B. Galerkin Turbochargers. p.650 (2010).

28. K.P. Seleznev, Yu.B. Galerkin Centrifugal compressors p. 271 (1982)

29. V.F. Ris Centrifugal compressor machines. 351 p. (1981)

30. G.N. Den Mechanics of flow in centrifugal compressors 268 p. (1973)

31. C.P. Livshits Aerodynamics of centrifugal compressor machines 340 p. (1966)

32. Yu.B. Galerkin, A.F. Rekstin, K.V. Soldatova, A.A. Drozdov Compressors and pneumatics. 7 p. 32-43 (2016)

33. A.F. Rekstin, Yu.B. Galerkin Bulletin Perm National Research Polytechnic University. 20(2). p. 43-54. DOI: 10.15593/2224-9877/2018.2.06 (2018)

34. A.V. Grigoriev, K.V. Solovyov, Yu.B. Galerkin, A.F. Rekstin Compressors and pneumatics. 6 p. 21-27 (2014)

35. A.F. Rekstin, K.V. Soldatova, A.A. Drozdov Klimov readings. Perspective directions of the aircraft engine structure. p. 45-55 (2013)

36. Yu. Galerkin, A. Rekstin, K. Soldatova International Conference on Numerical Methods in Industrial Processes. Vol: 9 No: 01 (2015)

37. A.N. Karpov Dis ... Cand. tech. Sciences 137 p. (2011) 